\title{
Angiotensin-converting enzyme inhibitors and angiotensin-II receptor blockers: do future research needs differ across disease states?
}

This article was published in the following Dove Press journal:

Comparative Effectiveness Research

9 January 2013

Number of times this article has been viewed

\author{
Matthew J Crowley ${ }^{1,2}$ \\ Benjamin J Powers ${ }^{1,2}$ \\ Evan R Myers ${ }^{1,3}$ \\ Douglas C McCrory' \\ Amanda J McBroom' \\ Gillian D Sanders' \\ 'Duke Evidence-Based Practice \\ Center, Duke Clinical Research \\ Institute, ${ }^{2}$ Center for Health Service \\ Research, Durham VA Medical Center, \\ ${ }^{3}$ Department of Obstetrics and \\ Gynecology, Duke University Medical \\ Center, Durham, NC, USA
}

Background: The purpose of this study was to compare the findings of two Agency for Healthcare Research and Quality (AHRQ) future research need prioritization projects: 1) future research needs pertaining to comparative effectiveness of angiotensin-converting enzyme inhibitors (ACE-I) and angiotensin-II receptor blockers (ARB) in ischemic heart disease (IHD); and 2) future research needs pertaining to comparative effectiveness of ACE-I/ ARB in hypertension.

Methods: Each of the two future research need prioritization projects was based on an AHRQsponsored comparative effectiveness review. For each project, we worked with the authors of the corresponding comparative effectiveness review to identify evidence gaps pertaining to the comparative effectiveness of ACE-I/ARB in the target condition, and performed an update of each review's literature search strategy. We then formed a distinct stakeholder group for each future research need prioritization project and engaged each group in sequential exercises to rank the identified evidence gaps.

Results: Although these projects engaged distinct stakeholder groups and relied upon different evidence bases, we noted near-complete overlap between the highest priority evidence gaps identified for each project. Stakeholders prioritized research: facilitating tailored treatment based on patient characteristics (eg, impact of demographics and comorbidities on ACE-I/ ARB effectiveness); targeting optimal implementation of ACE-I/ARB among patients most likely to benefit (eg, studies to improve evidence-based use, treatment adherence, and quality of life with ACE-I/ARB); and examining the impact of ACE-I/ARB on the incidence of new cardiovascular/metabolic diagnoses.

Conclusion: Designing studies to address high priority evidence gaps for multiple disease conditions simultaneously may facilitate future comparisons of ACE-I/ARB, and may likewise be an appropriate strategy to improve research efficiency in other comparative effectiveness research scenarios.

Keywords: comparative effectiveness research, future research needs, hypertension, ischemic heart disease

\section{Introduction}

Comparative effectiveness research is central to health care reform efforts intended to improve patient outcomes and reduce long-term costs. ${ }^{1}$ Recently, there has been substantial federal investment in comparative effectiveness research, including via the American Recovery and Reinvestment Act and the Affordable Care Act, the latter of which set aside funding to create a comparative effectiveness infrastructure with the Patient Centered Outcomes Research Institute. ${ }^{2}$ Establishing high priority areas for future investigation is integral to comparative effectiveness research. ${ }^{1}$
Correspondence: Matthew J Crowley HSR\&D I52, 508 Fulton Street, Durham, NC 27705, USA

$\mathrm{Tel}+1919684811 \mathrm{I}$

$\mathrm{Fax}+19194165836$

Email matthew.crowley@dm.duke.edu 
The Agency for Healthcare Research and Quality (AHRQ) Effective Health Care Program uses rigorous comparative effectiveness reviews to synthesize existing comparative effectiveness evidence for different health care interventions. ${ }^{3}$ The AHRQ has also developed a systematic process to identify gaps in the comparative effectiveness evidence base, which utilizes stakeholder input to prioritize evidence gaps into clearly defined "future research needs" ${ }^{4}$ Future research need prioritization projects aim to identify high priority research areas, thereby maximizing the efficiency with which researchers and funders can address gaps in the comparative effectiveness research evidence base. ${ }^{3}$

Angiotensin-converting enzyme inhibitors (ACE-I) and angiotensin II receptor blockers (ARB) are key pharmacotherapy agents for disorders such as ischemic heart disease and hypertension. Because the choice between these two medication classes is common and relevant for both clinicians and policymakers, AHRQ recently sponsored two separate comparative effectiveness reviews focusing on the use of ACE-I/ARB in ischemic heart disease and hypertension. ${ }^{5,6}$ According to these comparative effectiveness reviews, ACE-I reduced mortality and cardiovascular events relative to placebo among patients with ischemic heart disease, ${ }^{5}$ but increased syncope and cough; ARB reduced a composite of cardiovascular mortality, nonfatal myocardial infarction, and stroke relative to placebo, but fewer data were available than for ACE-I. No studies directly compared ACE-I and ARB in patients with ischemic heart disease. In patients with hypertension, ${ }^{6} \mathrm{ACE}-\mathrm{I}$ and ARB did not have differential impacts on blood pressure, mortality, cardiovascular events, quality of life, or intermediate outcomes (lipids, diabetes control, and renal function). ACE-I were associated with higher rates of cough and medication discontinuation due to adverse effects, but no differences in the effect of medications on treatment adherence or angioedema were seen.

AHRQ supported the Duke Evidence-based Practice Center in conducting two future research need prioritization projects corresponding to the ischemic heart disease and hypertension comparative effectiveness reviews. These two projects sought to engage stakeholders to rank the evidence gaps identified during each comparative effectiveness review systematically in terms of importance for future research. ${ }^{78}$ The purpose of the current report is to compare our findings regarding future research need prioritization with respect to the comparative effectiveness of ACE-I/ARB in ischemic heart disease and hypertension, and in so doing, highlight a potential strategy to increase the efficiency with which comparative effectiveness reviews can address high priority evidence gaps.

\section{Materials and methods}

The future research need prioritization processes utilized for the ischemic heart disease (2010) and hypertension (2012) projects were similar and are described in greater detail elsewhere. ${ }^{7,8}$ For each project, our Duke Evidence-based Practice Center worked with the authors of the corresponding comparative effectiveness review to create an initial list of evidence gaps pertaining to the comparative effectiveness of ACE-I/ARB in the target condition. To inform each prioritization process, we then performed an update of the literature search strategy for each of the comparative effectiveness reviews, as well as a horizon scan for active and recently concluded studies relevant to the identified evidence gaps. Of note, the two comparative effectiveness reviews evaluated nonoverlapping sets of studies pertaining to their respective target condition.

We formed a stakeholder group for each project; these groups included experts in the content area, health care policymakers, and representatives from funding agencies, generalist and specialty professional societies, and patient groups. Each stakeholder completed a disclosure statement, was screened for conflicts of interest, and was approved by AHRQ. For both projects, we elicited stakeholder input to expand our initial lists of evidence gaps. We then engaged the stakeholder groups in sequential exercises to rank the identified evidence gaps. These exercises utilized conference calls to: describe our prioritization methods; ${ }^{3}$ discuss findings from the corresponding comparative effectiveness review; share the literature review updates conducted by our Duke Evidence-based Practice Center; and facilitate group discussion. The final future research need rankings for each project were determined by tallying each stakeholder's final individual ranking. Investigators did not participate in the ranking process, but did moderate group discussions to assure that all stakeholders had the chance to ask questions and express views. An AHRQ task order officer participated in all teleconferences and was included in all communications with stakeholders. Online survey applications were used to solicit and compile future research need rankings for individual stakeholders.

For this report, we compare the highest priority future research needs identified during the ischemic heart disease and hypertension projects. Because there was some overlap between the stakeholder groups for these projects (two of 17 unique stakeholders), we conducted a sensitivity analysis for 
our comparison between the two projects by re-examining the prioritized future research needs for both projects without input from these individuals.

\section{Results}

\section{Identification of future research needs}

Based upon input from comparative effectiveness review investigators and stakeholders for each project, we generated separate lists of potential future research needs pertaining to the use of ACE-I/ARB in ischemic heart disease and hypertension. In all, 16 evidence gaps were identified for the ischemic heart disease project and 25 for the hypertension project. Complete evidence gap lists are shown in Appendix A, Table A1.

\section{Stakeholder groups}

The ischemic heart disease project engaged nine stakeholders and the hypertension project engaged 10 stakeholders. Two individuals were included in both stakeholder groups.

\section{Future research need prioritization}

The highest ranked future research needs for the ischemic heart disease and hypertension projects are shown in Figure 1 (full prioritized lists in Appendix B, Tables B1 and B2). We noted substantial overlap between the high-priority future research needs for the two projects, such that the top six future research needs for ischemic heart disease were represented among the seven highest priority hypertension future research needs. The sole evidence gap that did not overlap between the two projects was the hypertension evidence gap, ie, "studies of cardiovascular/cerebrovascular events compared across ACE-I/ARB'. The analogous evidence gap for the ischemic heart disease project, "impact of ACE-I/ARB on cardiovascular outcomes," was ranked ninth of 16 .

\section{Sensitivity analysis}

In order to evaluate whether the input of the two stakeholders included in both groups might have contributed to the observed similarity in the results of these projects, we excluded these stakeholders' individual rankings and recalculated the final future research need rankings for each project. The highest priority future research needs did not change substantially for either project following this exercise. For the ischemic heart disease project, the third-ranked future research needs, "comorbidities," switched places with the

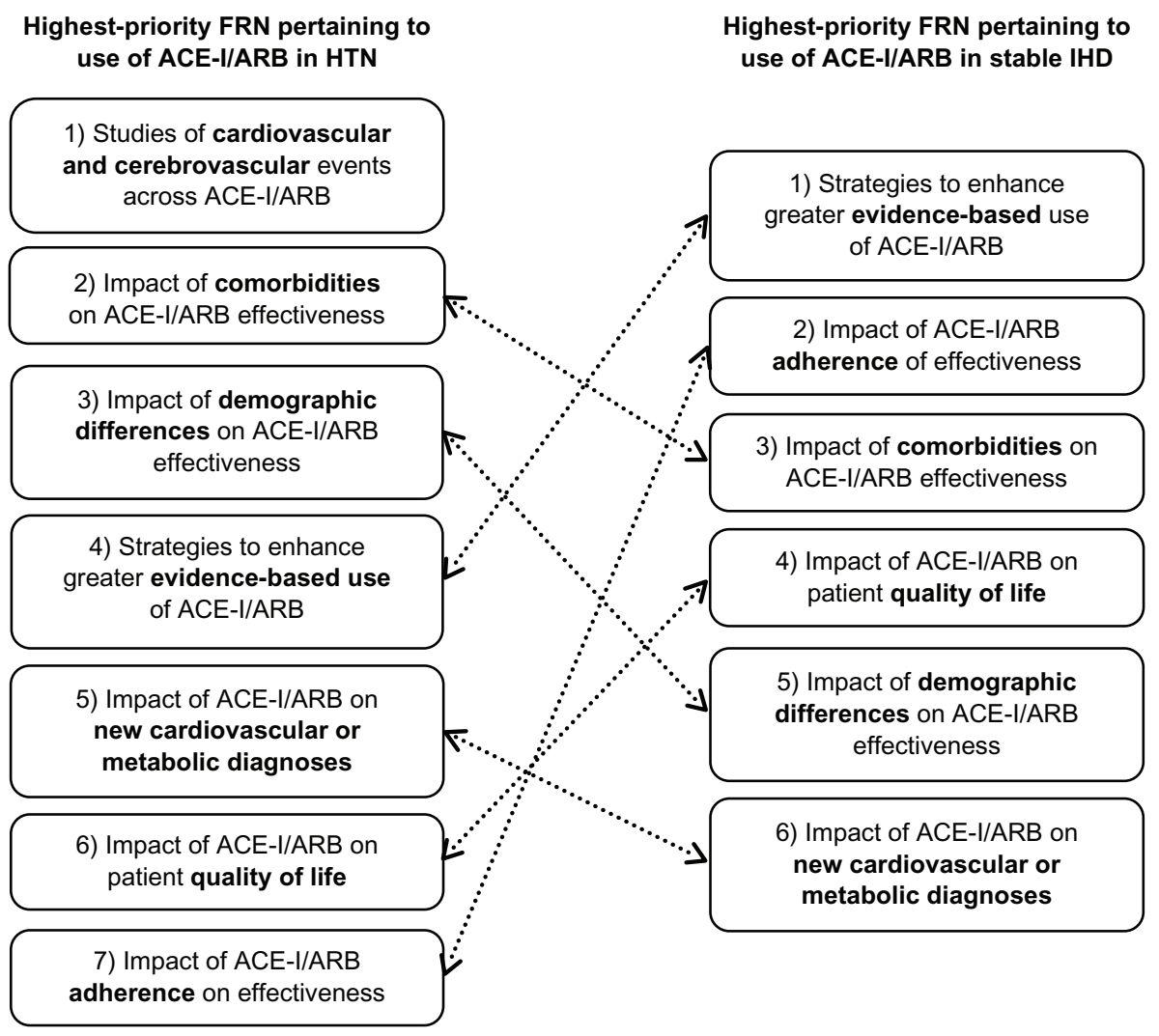

Figure I Overlap between high priority evidence gaps identified in two future research needs prioritization projects.

Abbreviations: ACE-I, angiotensin-converting enzyme inhibitors; ARB, angiotensin II receptor blockers/antagonists; FRN, future research need; HTN, hypertension; IHD, ischemic heart disease. 
fourth-ranked future research need "quality of life." For the hypertension project, the fifth-ranked future research need, "new cardiovascular/metabolic diagnoses," switched places with the sixth-ranked future research need, "quality of life," and the seventh-ranked future research need, "adherence" dropped into a tie with the subsequent three future research needs (Appendix B).

\section{Discussion}

Establishing high priority areas for future investigation is essential to maximizing the impact and efficiency of comparative effectiveness research. We conducted separate projects designed to prioritize future research pertaining to the comparative effectiveness of ACE-I/ARB in two different target conditions, ie, ischemic heart disease and hypertension. ${ }^{7,8}$ Although these projects engaged distinct stakeholder groups and evaluated different bodies of literature, we noted nearcomplete overlap between the highest priority evidence gaps identified for each disease condition. Stakeholders prioritized: research facilitating tailored treatment based on individual patient characteristics (eg, the impact of demographics and comorbidities on the effectiveness of ACE-I/ARB); research designed to optimize implementation of these medications among patients most likely to benefit (eg, studies to improve evidence-based use, treatment adherence, and patient quality of life with $\mathrm{ACE}-\mathrm{I} / \mathrm{ARB}$ ); and research examining the impact of ACE-I/ARB on the incidence of new cardiovascular/ metabolic diagnoses.

One future research need, "studies of cardiovascular/cerebrovascular events," was ranked highly for the hypertension but not the ischemic heart disease project. This may relate to a traditional focus on cardiovascular outcomes in ischemic heart disease studies, which has resulted in extensive outcome data. In contrast, hypertension studies have focused on blood pressure control as a primary outcome, so cardiovascular/ cerebrovascular event data pertaining to this condition are sparser. Because conditions like hypertension and ischemic heart disease tend to co-occur among patients receiving ACE-I/ARB, it may be reasonable to extrapolate information from studies reporting cardiovascular/cerebrovascular outcomes in ischemic heart disease when weighing this future research need among patients with hypertension. ${ }^{9}$

Redundancy in comparative effectiveness research for ACE-I/ARB has previously been highlighted as an example of research inefficiency. ${ }^{9}$ Because the high-priority evidence gaps we identified for these two future research need prioritization projects are so similar, a combined study of ACE-I/ $\mathrm{ARB}$ in hypertension and ischemic heart disease, either via pooling existing data or designing new studies that cross the two disease conditions, may increase the efficiency with which these gaps can be addressed. Combining data across target conditions may be especially powerful in evaluating future research needs that are not disease-specific, such as evidence-based use, treatment adherence, and patient quality of life, or future research needs pertaining to rare events, such as the incidence of new cardiovascular or metabolic diagnoses with ACE-I/ARB.

\section{Limitations}

Although our sensitivity analysis indicates that the final future research need rankings did not change substantially with removal of the two overlapping stakeholders' input, it is possible that these stakeholders' participation in conference calls for both projects contributed to the observed similarity between the results of the projects. However, because the final prioritized future research need lists were based on stakeholders' individual rankings and not on group consensus, we feel it is unlikely that the two overlapping stakeholders exerted undue influence on the other participants. It is likewise possible that our Duke Evidence-based Practice Center's moderation of conference calls for both projects unintentionally influenced the future research need rankings for the two projects, but because the investigators did not participate in the future research need ranking process, this likelihood is small.

In summary, we conducted two separate projects designed to prioritize future research needs pertaining to the comparative effectiveness of ACE-I/ARB in ischemic heart disease and hypertension, and noted almost total overlap in the results. Our findings suggest that designing studies to address shared future research needs simultaneously may facilitate future comparative effectiveness research of ACE-I/ $\mathrm{ARB}$ in hypertension and ischemic heart disease. In order to help assure that future research need prioritization serves its primary purpose, ie, maximizing the efficiency with which gaps in the comparative effectiveness evidence base can be addressed, researchers and funders should remain vigilant for other opportunities to address evidence gaps simultaneously across multiple disease conditions.

\section{Acknowledgment}

This project was funded under Contract No. 290-200710066-I from AHRQ, U.S. Department of Health and Human Services. The authors of this report are responsible for its content. Statements in the report should not be construed as endorsement by the AHRQ or the U.S. Department of Health and Human Services. 


\section{Disclosure}

The authors report no conflicts of interest in this work.

\section{References}

1. Conway PH, Clancy C. Comparative-effectiveness research implications of the Federal Coordinating Council's Report. $N$ Engl J Med. 2009;361(4):328-330.

2. Selby JV, Beal AC, Frank L. The Patient-Centered Outcomes Research Institute (PCORI) national priorities for research and initial research agenda. JAMA. 2012;307(15):1583-1584.

3. Agency for Healthcare Research and Quality. Future research needs methods research series. Available at: http://www.effectivehealthcare. ahrq.gov/index.cfm/search-for-guides-reviews-and-reports/?pageactio $\mathrm{n}=$ displayProduct\&productID=481. Accessed October 2, 2012.

4. Chang SM, Carey T, Kato EU, Guise JM, Sanders GD. Identifying research needs for improving health care. Ann Intern Med. 2012;157(6): 439-445.

5. Coleman CI, Baker WL, Kluger J, et al. Comparative effectiveness of angiotensin converting enzyme inhibitors or angiotensin II receptor blockers added to standard medical therapy for treating stable ischemic heart disease. Rockville, MD: Agency for Healthcare Research and Quality. Oct 2009. Available at: http://www.effectivehealthcare.ahrq. gov/reports/final.cfm. Accessed September 3, 2012.
6. Sanders GD, Coeytaux R, Dolor RJ, et al. Angiotensin-converting enzyme inhibitors (ACEIs), angiotensin II receptor antagonists (ARBs), and direct renin inhibitors for treating essential hypertension: an update. Comparative Effectiveness Review No 34. AHRQ Publication No 11-EHC063-EF Rockville, MD: Agency for Healthcare Research and Quality. Jun 2011 Available at: http://www.effectivehealthcare.ahrq.gov/reports/final.cfm. Accessed September 3, 2012.

7. Sanders GD, Powers B, Crowley M, et al. Future research needs for angiotensin converting enzyme inhibitors or angiotensin II receptor blockers added to standard medical therapy for treating stable ischemic heart disease. Future Research Needs Paper No 8. AHRQ Publication No 11-EHC006-EF. Rockville, MD: Agency for Healthcare Research and Quality. Nov 2010. Available at: http://www.effectivehealthcare. ahrq.gov/reports/final.cfm. Accessed September 3, 2012.

8. Powers BJ, Crowley MJ, McCrory DC, et al. Future research needs for comparing angiotensin-converting enzyme inhibitors (ACEIs), angiotensin II receptor antagonists (ARBs), and direct renin inhibitors (DRIs) in the treatment of hypertension. AHRQ Publication No 12-EHC046-EF. Rockville, MD: Agency for Healthcare Research and Quality; Mar 2012. Available at: http://www.effectivehealthcare.ahrq.gov/reports/final.cfm. Accessed September 3, 2012.

9. Powers BJ, Coeytaux RR, Dolor RJ, et al. Updated report on comparative effectiveness of ACE inhibitors, ARBs, and direct renin inhibitors for patients with essential hypertension: much more data, little new information. J Gen Intern Med. 2012;27(6):716-729. 


\section{Appendices}

\section{Appendix A}

Table AI Complete lists of identified evidence gaps for two future research need prioritization projects pertaining to the comparative effectiveness of ACE-I and ARB in hypertension and ischemic heart disease (plus DRI for hypertension), in PICO format

\section{Future research needs in ischemic heart disease}

Population

- Impact of comorbidities (such as hypertension, CHF with or without preserved LV function, diabetes, peripheral arterial disease, chronic kidney disease, prior coronary revascularization, single-vessel versus multivessel coronary artery disease) on ACE-I/ARB effectiveness or harms in patients with stable ischemic heart disease

- Impact of demographic differences (such as age, race, gender) on ACE-I/ARB effectiveness or harms in patients with stable ischemic heart disease

- Impact of concurrent medications (such as antiplatelet agents, lipid-lowering medications, other antihypertensives) on ACE-I/ARB effectiveness or harms in patients with stable ischemic heart disease

- Impact of genetic differences (such as angiotensin-converting enzyme or angiotensin II receptor gene polymorphisms) on ACE-I/ARB effectiveness or harms in patients with stable ischemic heart disease

Intervention

- Strategies to enhance greater evidence-based use of ACE-I/ARB

- Impact of ACE-I/ARB adherence (including differential adherence within and between medication classes) on their effectiveness or harms in patients with stable ischemic heart disease

- Impact of dose response (impact of medication dose or dosing interval) of ACE-I and ARB on their effectiveness or harms in patients with stable ischemic heart disease

- Impact of class effect (impact of differences between specific agents within each class) of ACE-I and ARB on their effectiveness or harms in patients with stable ischemic heart disease

Comparator

- The benefit of ACE-I/ARB relative to alternative medication classes (calcium channel blocker, diuretic, or beta-blocker) with respect to their effectiveness or harms in patients with stable ischemic heart disease

Outcome

- Impact of ACE-I/ARB on incidence of new diagnoses (such as diabetes, atrial fibrillation, CHF with or without preserved LV function) in patients with stable ischemic heart disease

- Impact of ACE-I/ARB on quality of life in patients with stable ischemic heart disease

- Impact of ACE-I/ARB on cardiovascular outcomes (such as cardiovascular death, nonfatal MI, CVA, hospitalization for CHF, and surrogates such as blood pressure control and measures of atherosclerosis) in patients with stable ischemic heart disease

- Impact of ACE-I/ARB on utilization and cost of therapy in patients with stable ischemic heart disease

- Impact of ACE-I/ARB on progression of renal insufficiency or development of dialysis dependence in patients with stable ischemic heart disease

- Impact of ACE-I/ARB on development of nonangioedema adverse effects (such as hypotensive symptoms, cough, syncope, diarrhea, renal insufficiency, hyperkalemia) in patients with stable ischemic heart disease

- Impact of ACE-I/ARB on development of angioedema in patients with stable ischemic heart disease.

Future research needs in hypertension

Population

- Impact of comorbidities (such as ischemic heart disease, CHF with or without preserved LV function, diabetes, peripheral arterial disease, chronic kidney disease, prior coronary revascularization; single-vessel versus multivessel coronary artery disease) on ACE-I/ARB/DRI effectiveness or harms in patients with hypertension

- Impact of demographic differences (such as age, race, gender) on ACE-I/ARB/DRI effectiveness or harms in patients with hypertension

- Impact of genetic differences (such as angiotensin-converting enzyme or angiotensin II receptor gene polymorphisms) on ACE-I/ARB/DRI effectiveness or harms

- Impact of health risk behaviors such as diet, exercise, smoking, alcohol intake on ACE-I/ARB/DRI effectiveness or harms Intervention and comparator

- Impact of dose response (impact of medication dose or dosing interval) of ACE-I/ARB/DRI on their effectiveness or harms

- Differential impact of specific agents or subclasses (based on tissue specificity, chemical properties, or pharmacokinetics) within each medication class on ACE-I/ARB/DRI effectiveness or harms

- Benefit of ACE-I/ARB/DRI relative to alternative medication classes (calcium channel blocker, diuretic, or beta-blocker) with respect to their effectiveness or harms

- Impact of concurrent medications (such as antiplatelet agents, lipid-lowering medications, diuretics, other antihypertensives) on ACE-I/ARB/DRI effectiveness or harms 
Table Al (Continued)

- Impact of ACE-I/ARB/DRI monotherapy compared with ACE-I, ARB, and/or DRI combination therapy in essential hypertension

- Impact of ACE-I/ARB/DRI alone compared with ACE-I/ARB/DRI combined with aldosterone receptor antagonists

- Impact of $A C E-I / A R B / D R I$ alone compared with ACE-I/ARB/DRI in combination with a diuretic

Outcome

- Studies of cardiovascular and cerebrovascular events compared across the three medication classes requiring evaluation of outcomes over several years

- Evaluation of cancer-related outcomes, which are infrequently reported in the existing literature

- Evaluation of the incidence, timing, and clinical consequences of angioedema in patients treated with ACE-I, ARB, or DRI

- Impact of ACE-I/ARB/DRI on incidence of new cardiovascular or metabolic diagnoses (such as diabetes, atrial fibrillation, $\mathrm{CHF}$ with or without preserved LV function)

- Impact of ACE-I/ARB/DRI on patient health status, including quality of life and functional capacity

- Impact of ACE-I/ARB/DRI on utilization and cost of therapy

- Impact of ACE-I/ARB/DRI on progression of renal insufficiency or development of dialysis dependence

- Impact of ACE-I/ARB/DRI on development of nonangioedema adverse effects (such as hypotensive symptoms, cough, syncope, diarrhea, renal insufficiency, hyperkalemia)

- Impact of changing trends in outcome event rates over time on comparative effectiveness of ACE-I, ARB, and DRI

Other

Implementation gaps

- Relative medication adherence and persistence with drug therapy across the different classes of drugs

- Studies of the impact of health system financing, delivery, and/or organizational interventions on evidence based medication prescribing and patient adherence

- Practical clinical trials or other external validity-oriented studies that compare these medications in practice settings that better represent realworld practice

Methods for evidence synthesis

- Methods for synthesis of data across clinical conditions (eg, CHF, ischemic heart disease, and chronic kidney disease) to understand the comparative effectiveness of ACE-I, ARB, and DRI better.

- Methods for individual patient data meta-analysis, to examine subgroups better in the absence of other confounders

Abbreviations: ACE-I, angiotensin-converting enzyme inhibitors; ARB, angiotensin II receptor blockers/antagonists; CHF, congestive heart failure; CVA, cerebrovascular accident; IHD, ischemic heart disease; LV, left ventricular; MI, myocardial infarction; PICO, population, interventions, comparators, and outcomes; DRI, direct renin inhibitor. 


\section{Appendix B}

Prioritized lists of evidence gaps for two future research need prioritization projects pertaining to the comparative effectiveness of ACE-I and ARB in hypertension and ischemic heart disease.

Table B I Future research needs in ischemic heart disease

\begin{tabular}{|c|c|}
\hline Ranking & Research area \\
\hline & Top tier \\
\hline I & Strategies to enhance greater evidence-based use of ACE-I/ARB \\
\hline 2 & $\begin{array}{l}\text { Impact of ACE-I/ARB adherence (including differential adherence within and between medication classes) on their } \\
\text { effectiveness or harms in patients with stable IHD }\end{array}$ \\
\hline 3 & $\begin{array}{l}\text { Impact of comorbidities (such as hypertension, CHF with or without preserved LV function, diabetes, peripheral arterial } \\
\text { disease, chronic kidney disease, prior coronary revascularization, single-vessel versus multivessel coronary artery disease) on } \\
\text { ACE-I/ARB effectiveness or harms in patients with stable IHD }\end{array}$ \\
\hline 4 & Impact of ACE-I/ARB on patient quality of life in patients with stable IHD \\
\hline 5 & $\begin{array}{l}\text { Impact of demographic differences (such as age, race, gender) on ACE-I/ARB effectiveness or harms in patients with stable } \\
\text { IHD }\end{array}$ \\
\hline 6 & $\begin{array}{l}\text { Impact of ACE-I/ARB on incidence of new diagnoses (such as diabetes, atrial fibrillation, CHF with or without preserved LV } \\
\text { function) in patients with stable IHD } \\
\text { Lower tier }\end{array}$ \\
\hline 7 & $\begin{array}{l}\text { Benefit of ACE-I/ARB relative to alternative medication classes (calcium channel blocker, diuretic, or beta-blocker) with } \\
\text { respect to their effectiveness or harms in patients with stable IHD }\end{array}$ \\
\hline 8 & Impact of ACE-I/ARB on utilization and cost of therapy in patients with stable IHD \\
\hline 9 & $\begin{array}{l}\text { Impact of ACE-I/ARB on cardiovascular outcomes (such as cardiovascular death, nonfatal MI, CVA, hospitalization for CHF, } \\
\text { and surrogates such as blood pressure control, measures of atherosclerosis) in patients with stable IHD }\end{array}$ \\
\hline 10 & $\begin{array}{l}\text { Impact of concurrent medications (such as antiplatelet agents, lipid-lowering medications, other antihypertensives) on ACE-I/ } \\
\text { ARB effectiveness or harms in patients with stable IHD }\end{array}$ \\
\hline 11 & Impact of ACE-I/ARB on progression of renal insufficiency or development of dialysis dependence in patients with stable IHD \\
\hline 12 & $\begin{array}{l}\text { Impact of genetic differences (such as angiotensin-converting enzyme or angiotensin II receptor gene polymorphisms) on } \\
\text { ACE-I/ARB effectiveness or harms in patients with stable IHD }\end{array}$ \\
\hline 13 & $\begin{array}{l}\text { Impact of dose response (impact of medication dose or dosing interval) of ACE-I and ARB on their effectiveness or harms in } \\
\text { patients with stable IHD }\end{array}$ \\
\hline 14 & $\begin{array}{l}\text { Impact of class effect (impact of differences between specific agents within each class) of ACE-I and ARB on their effectiveness } \\
\text { or harms in patients with stable IHD }\end{array}$ \\
\hline 15 & $\begin{array}{l}\text { Impact of ACE-I/ARB on development of nonangioedema adverse effects (such as hypotensive symptoms, cough, syncope, } \\
\text { diarrhea, renal insufficiency, hyperkalemia) in patients with stable IHD }\end{array}$ \\
\hline 16 & Impact of ACE-I/ARB on development of angioedema in patients with stable IHD \\
\hline
\end{tabular}

Abbreviations: ACE-I, angiotensin-converting enzyme inhibitors; ARB, angiotensin II receptor blockers/antagonists; CHF, congestive heart failure; CVA, cerebrovascular accident; IHD, ischemic heart disease; LV, left ventricular; MI, myocardial infarction. 
Table B2 Future research needs in hypertension

\begin{tabular}{|c|c|}
\hline Ranking & Research area \\
\hline & Top tier \\
\hline I & $\begin{array}{l}\text { Studies of cardiovascular and cerebrovascular events compared across the three medication classes, requiring evaluation of } \\
\text { outcomes over several years }\end{array}$ \\
\hline 2 & $\begin{array}{l}\text { Impact of comorbidities (such as ischemic heart disease, CHF with or without preserved LV function, diabetes, peripheral } \\
\text { arterial disease, chronic kidney disease, prior coronary revascularization; single-vessel versus multivessel coronary artery } \\
\text { disease) on ACE-I/ARB/DRI effectiveness or harms in patients with hypertension }\end{array}$ \\
\hline 3 & $\begin{array}{l}\text { Impact of demographic differences (such as age, race, gender) on ACE-I/ARB/DRI effectiveness or harms in patients with } \\
\text { hypertension }\end{array}$ \\
\hline 4 & $\begin{array}{l}\text { Practical clinical trials or other external validity-oriented studies that compare these medications in practice settings that better } \\
\text { represent real-world practice. }\end{array}$ \\
\hline 5 & $\begin{array}{l}\text { Impact of ACE-I/ARB/DRI on incidence of new cardiovascular or metabolic diagnoses (such as diabetes, atrial fibrillation, CHF } \\
\text { with or without preserved LV function) }\end{array}$ \\
\hline 6 & Impact of ACE-I/ARB/DRI on patient health status including quality of life and functional capacity \\
\hline 7 & $\begin{array}{l}\text { Relative medication adherence and persistence with drug therapy across the different classes of drugs. } \\
\text { Middle tier }\end{array}$ \\
\hline 8 & $\begin{array}{l}\text { Benefit of ACE-I/ARB/DRI relative to alternative medication classes (calcium channel blocker, diuretic, or beta-blocker) with } \\
\text { respect to their effectiveness or harms }\end{array}$ \\
\hline 9 & Impact of $A C E-I / A R B / D R I$ on utilization and cost of therapy \\
\hline 10 & Impact of health risk behaviors, such as diet, exercise, smoking, and alcohol intake, on ACE-I/ARB/DRI effectiveness or harms \\
\hline II & Impact of ACE-I/ARB/DRI on progression of renal insufficiency or development of dialysis dependence \\
\hline 12 & Impact of changing trends in outcome event rates over time on the comparative effectiveness of ACE-I, ARB, and DRI \\
\hline 13 & Better understanding of provider prescribing patterns and interventions to support evidence-based decision-making \\
\hline 14 & $\begin{array}{l}\text { Impact of class effect (impact of differences between specific agents within each class) of ACE-I/ARB/DRI on their effectiveness } \\
\text { or harms }\end{array}$ \\
\hline 15 & $\begin{array}{l}\text { Impact of ACE-I/ARB/DRI on development of nonangioedema adverse effects (such as hypotensive symptoms, cough, syncope, } \\
\text { diarrhea, renal insufficiency, hyperkalemia) }\end{array}$ \\
\hline & Lower tier \\
\hline 16 & $\begin{array}{l}\text { Impact of concurrent medications (such as antiplatelet agents, lipid-lowering medications, diuretics, other antihypertensives) on } \\
\text { ACE-I/ARB/DRI effectiveness or harms }\end{array}$ \\
\hline 17 & $\begin{array}{l}\text { Methods for synthesis of data across clinical conditions (eg, CHF, ischemic heart disease, and chronic kidney disease) to } \\
\text { understand better the comparative effectiveness of ACE-I, ARB, and DRI }\end{array}$ \\
\hline 18 & Methods for individual patient data meta-analysis, to examine subgroups better in the absence of other confounders \\
\hline 19 & Impact of dose response (impact of medication dose or dosing interval) of ACE-I/ARB/DRI on their effectiveness or harms \\
\hline 20 & $\begin{array}{l}\text { Impact of genetic differences (eg, angiotensin-converting enzyme or angiotensin II receptor gene polymorphisms) on ACE-I/ } \\
\text { ARB/DRI effectiveness or harms }\end{array}$ \\
\hline 21 & Impact of ACE-I, ARB, or DRI monotherapy compared with ACE-I, ARB, and/or DRI combination therapy in hypertension \\
\hline 22 & Impact of ACE-I/ARB/DRI alone compared with ACE-I/ARB/DRI combined with aldosterone receptor antagonists \\
\hline 23 & Impact of ACE-I/ARB/DRI alone compared with ACE-I/ARB/DRI in combination with a diuretic \\
\hline 24 & Evaluation of cancer-related outcomes, which are infrequently reported in the existing literature \\
\hline 25 & Evaluation of incidence, timing, and clinical consequences of angioedema in patients treated with ACE-I, ARB, or DRI \\
\hline
\end{tabular}

Abbreviations: ACE-I, angiotensin-converting enzyme inhibitor; ARB, angiotensin II receptor blocker/antagonist; CHF, congestive heart failure; DRI, direct renin inhibitor; LV, left ventricular.

\section{Publish your work in this journal}

Comparative Effectiveness Research is an international, peer reviewed open access journal focusing on comparative effectiveness of health care including preventative health care strategies, diagnostic strategies, diagnostic technology, medical devices, drugs, medical technology, health systems and organization. The manuscript management system is completely online and includes a very quick and fair peer-review system. Visit http://www.dovepress.com/testimonials.php to read real quotes from published authors. 